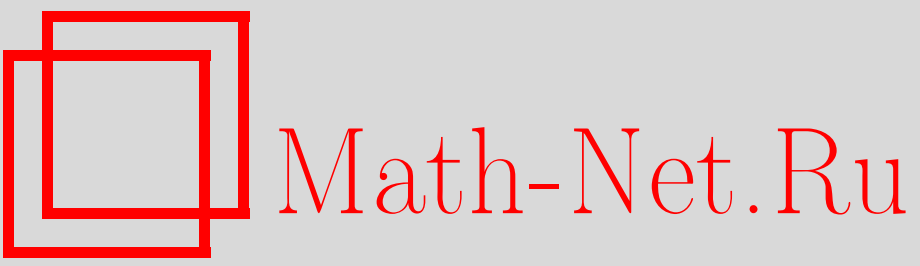

М. Х. Шерматов, О точечном взаимодействии двух фермионов и одной частицы иной природы, ТМФ, 2003, том 136, номер 2, 257-270

DOI: https://doi.org/10.4213/tmf226

Использование Общероссийского математического портала Math-Net.Ru подразумевает, что вы прочитали и согласны с пользовательским соглашением

http://www.mathnet.ru/rus/agreement

Параметры загрузки:

IP : 54.224 .60 .19

26 апреля 2023 г., 08:59:11

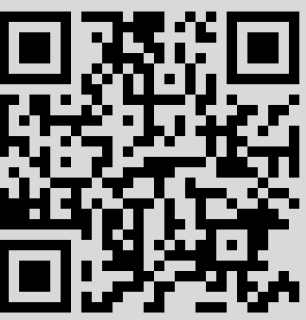




\section{О ТОЧЕЧНОМ ВЗАИМОДЕЙСТВИИ ДВУХ ФЕРМИОНОВ И ОДНОЙ ЧАСТИЦЫ ИНОЙ ПРИРОДЫ}

Рассматривается модель точечного взаимодействия двух фермионов и одной частицы иной природы, аналогичная модели Скорнякова-Тер-Мартиросяна и трактуемая с помощью теории самосопряженных расширений симметрических операторов. Показано, что если масса третьей частицы достаточно мала по сравнению с массой фермионов, то соответствующий оператор энергии имеет бесконечный набор связанных состояний со значениями энергии, уходящими на $-\infty$.

Ключевые слова: гамильтониан, точечное взаимодействие, симметрический оператор, расширение Скорнякова-Тер-Мартиросяна, самосопряженное расширение.

\section{1. ВВЕДЕНИЕ}

В работах Минлоса и Фаддеева [1], [2] впервые было предложено строгое математическое описание точечного взаимодействия трех одинаковых квантовых частиц (бозонов). Гамильтониан этой системы трактовался с помошью теории самосопряженных расширений симметрических операторов (см. [3]) и вводился как некоторое самосопряженное расширение симметрического оператора кинетической энергии трех частиц

$$
H_{0}=-\Delta_{x_{1}}-\Delta_{x_{2}}-\Delta_{x_{3}},
$$

определенного на области $D\left(H_{0}\right) \subset L_{2}^{s}\left(\left(\mathbb{R}^{3}\right)^{3}\right)$ функций трех переменных $x_{j} \in \mathbb{R}^{3}, j=$ $1,2,3$, обращающихся в нуль при совпадении любых двух аргументов $x_{j}=x_{k}, j \neq k$, $j, k=1,2,3$. Предложенное в работе [1] расширение оператора $H_{0}$ (обозначаемое через $H_{\varepsilon}, \quad \varepsilon \in \mathbb{R}^{1}$, и называемое расширением Скорнякова-Тер-Мартиросяна) является лишь симметрическим, но не самосопряженным. В работе [2] показано, что оператор $H_{\varepsilon}$ имеет индексы дефекта $(1,1)$, и построены все самосопряженные расширения $H_{\varepsilon, \beta}$ оператора $H_{\varepsilon}$. При этом оказалось, что эти расширения имеют не ограниченный снизу дискретный спектр. В работе [4] с использованием результатов работ [1], [2] изучался гамильтониан системы трех частищ (двух фермионов и одной частицы иной природы) с одинаковыми массами, взаимодействующих точечным образом. Этот гамильтониан вводился как расширение Скорнякова-Тер-Мартиросяна оператора $H_{0}$ в пространстве

* Самаркандское отделение АН Республики Узбекистан, Самарканд, Узбекистан. E-mail: mamarajab_2002@rambler.ru 
$L_{2}^{a(1,2)}\left(\left(\mathbb{R}^{3}\right)^{3}\right)$ функций, антисимметричных относительно переменных $x_{1}$ и $x_{2}$, и было показано, что эти расширения являются самосопряженными, полуограниченными и не имеют отрицательных собственных значений. В работе [5] обобшены результаты работ $[1],[2],[4]$ на случай трех различимых частищ (обладаюших даже различными массами).

В настояшей статье, следуя в основном схеме, использованной в работах [4], [5], мы рассмотрим систему трех частиц, состоящую из двух фермионов и одной частишы иной природы, взаимодействующих точечным образом. В отличие от работы [4] будем предполагать, что масса третьей частицы отличается от массы фермионов. Докажем, что если масса третьей частицы $m_{3}$ достаточно мала по сравнению с массой фермионов $m$, то расширение Скорнякова-Тер-Мартиросяна (обозначаемое через $H_{\varepsilon}, \varepsilon \in \mathbb{R}^{1}$ ) оператора $(2.1)$ в пространстве $L_{2}^{a(1,2)}\left(\left(\mathbb{R}^{3}\right)^{3}\right)$ не является самосопряженным и имеет ненулевые, равные и конечные индексы дефекта. В отличие от работ [1], [2], [5] в данной работе часть оператора $H_{\varepsilon}$ в подпространстве сферически-симметричных функций является самосопряженной, а его часть в подпространстве функций с угловым моментом $l=1$ симметрична и имеет индексы дефекта $(1,1)$. При этом все самосопряженные расширения оператора $H_{\varepsilon}$ имеют бесконечный набор собственных значений, уходяших на $-\infty$, и соответствуюшие собственные функции не являются сферически-симметричными.

\section{2. ПРЕДВАРИТЕЛЬНЫЕ СВЕДЕНИЯ И ВЫБОР РАСШИРЕНИЯ}

Гамильтониан системы двух фермионов и одной частицы иной природы, взаимодействуюших точечным образом, задается как некоторое самосопряженное расширение симметрического оператора

$$
H=-\frac{1}{2 m} \Delta_{x_{1}}-\frac{1}{2 m} \Delta_{x_{2}}-\frac{1}{2 m_{3}} \Delta_{x_{3}},
$$

действуюшего в пространстве $L_{2}^{a(1,2)}=\left\{\psi \in L_{2}\left(\left(\mathbb{R}^{3}\right)^{3}\right): \psi\left(x_{1}, x_{2}, x_{3}\right)=-\psi\left(x_{2}, x_{1}, x_{3}\right)\right\}$ волновых функций и определенного на области

$$
D(H)=\left\{\psi \in L_{2}^{a(1,2)}: \Delta_{x_{i}} \psi \in L_{2}^{a(1,2)}, \quad i=1,2,3,\left.\quad \psi\right|_{\Gamma_{j 3}}=0, \quad j=1,2\right\}
$$

где $\Gamma_{j 3} \subset\left(\mathbb{R}^{3}\right)^{3}$ - гиперплоскость $\Gamma_{j 3}=\left\{\left(x_{1}, x_{2}, x_{3}\right) \in\left(\mathbb{R}^{3}\right)^{3}: x_{j}=x_{3}\right\}$.

Далее удобно перейти к импульсному представлению. После такого перехода, отделения полного импульса $P=p_{1}+p_{2}+p_{3}$ и замены переменных $k_{j}=(\mathrm{m} / M) P-p_{j}$, $j=1,2, k_{3}=\left(m_{3} / M\right) P-p_{3}$ оператор $H$ принимает вид

$$
H=\frac{1}{2 M} P^{2}+\eta^{-1} h, \quad M=2 m+m_{3}, \quad \eta=\frac{2 m m_{3}}{m+m_{3}},
$$

и действует в тензорном произведении пространств $L_{2}\left(\mathbb{R}^{3}\right) \otimes L_{2}^{a}\left(\left(\mathbb{R}^{3}\right)^{2}\right)$, где $L_{2}^{a}\left(\left(\mathbb{R}^{3}\right)^{2}\right)=$ $\left\{f \in L_{2}\left(\left(\mathbb{R}^{3}\right)^{2}\right): f\left(k_{1}, k_{2}\right)=-f\left(k_{2}, k_{1}\right)\right\}$. В (2.3) самосопряженньй оператор $(1 / 2 M) P^{2}$ действует в $L_{2}\left(\mathbb{R}^{3}\right)$, а симметрический оператор $h$ действует в $L_{2}^{a}\left(\left(\mathbb{R}^{3}\right)^{2}\right)$ по формуле

$$
(h f)\left(k_{1}, k_{2}\right)=\left(k_{1}^{2}+\mu k_{1} k_{2}+k_{2}^{2}\right) f\left(k_{1}, k_{2}\right),
$$


где $\mu=2 m /\left(m+m_{3}\right), 0<\mu<2$. Область определения $D(h)$ оператора $h$ состоит из функций $f\left(k_{1}, k_{2}\right)$, удовлетворяюших условиям

$$
\int_{\mathbb{R}^{3}}\left|k_{1}^{2}+k_{2}^{2}\right|^{2}\left|f\left(k_{1}, k_{2}\right)\right|^{2} d k_{1} d k_{2}<\infty, \quad \int_{\mathbb{R}^{3}} f\left(k_{1}, k_{2}\right) d k_{j}=0, \quad j=1,2 .
$$

Поскольку оператор $h$ неотрицателен, естественно воспользоваться теорией расширений полуограниченных операторов (см. [6]). С помошью условия (2.4) нетрудно убедиться, что дефектное подпространство $\Re_{-1}(h)$ оператора $h+I$ состоит из элементов вида

$$
\Phi_{u}\left(k_{1}, k_{2}\right)=\frac{u\left(k_{1}\right)-u\left(k_{2}\right)}{k_{1}^{2}+\mu k_{1} k_{2}+k_{2}^{2}+1},
$$

где $u(k)$ - произвольная функция, удовлетворяющая условию

$$
\int_{\mathbb{R}^{3}}|u(k)|^{2}\left(k^{2}+1\right)^{-1 / 2} d k<\infty .
$$

Отсюда видно, что подпространство $\Re_{-1}(h)$ изоморфно гильбертову пространству $\Omega$ функций $u(k), k \in \mathbb{R}^{3}$, получающемуся пополнением $L_{2}\left(\mathbb{R}^{3}\right)$ по скалярному произведению $[u, v]=(K u, v)$, где $(\cdot, \cdot)$ - обычное скалярное произведение в $L_{2}\left(\mathbb{R}^{3}\right)$ и $K$ - положительный оператор в $L_{2}\left(\mathbb{R}^{3}\right)$, действующий по формуле

$$
(K u)(k)=\frac{2 \pi^{2} u(k)}{\sqrt{\left(4-\mu^{2}\right) k^{2} / 4+1}}-2 \int_{\mathbb{R}^{3}} \frac{u\left(k^{\prime}\right) d k^{\prime}}{k^{2}+\mu k k^{\prime}+\left(k^{\prime}\right)^{2}+1} .
$$

Лемма 1. Область определения $D\left(h^{*}\right)$ сопряхсенного оператора $h^{*}$ состоит из функиий вида

$$
g\left(k_{1}, k_{2}\right)=f\left(k_{1}, k_{2}\right)+\frac{u\left(k_{1}\right)-u\left(k_{2}\right)}{k_{1}^{2}+\mu k_{1} k_{2}+k_{2}^{2}+1}+\frac{v\left(k_{1}\right)-v\left(k_{2}\right)}{\left(k_{1}^{2}+\mu k_{1} k_{2}+k_{2}^{2}+1\right)^{2}},
$$

где $f \in D(h), \quad u, v \in \Omega$. Оператор $h^{*}$ действует на функиию вида (2.8) по формуле

$$
\left(h^{*} g\right)\left(k_{1}, k_{2}\right)=\left(k_{1}^{2}+\mu k_{1} k_{2}+k_{2}^{2}\right) g\left(k_{1}, k_{2}\right)-u\left(k_{1}\right)+u\left(k_{2}\right) .
$$

Лемма доказывается так же, как и аналогичная лемма 1 из работы [7].

Пусть $A$ - произвольный линейный оператор с плотной в $\Omega$ областью определения $D(A)$ и $h_{A}-$ сужение оператора $h^{*}$ на область

$$
D\left(h_{A}\right)=\left\{g \in D\left(h^{*}\right): g=f+\Phi_{u}+S_{1}^{-1} \Phi_{A u}, \quad f \in D(h), \quad u \in D(A)\right\},
$$

где $S_{1}$ - жесткое самосопряженное расширение оператора $h+I$. Для функций $g\left(k_{1}, k_{2}\right)$ из $D\left(h_{A}\right)$ в разложении $(2.8)$ выполняется соотношение вида

$$
v=A u, \quad u \in D(A) .
$$

Возьмем два произвольных элемента $g=f+\Phi_{u}+S_{1}^{-1} \Phi_{A u}$ и $g^{\prime}=f^{\prime}+\Phi_{u^{\prime}}+S_{1}^{-1} \Phi_{A u^{\prime}}$ из $D\left(h_{A}\right)$. Для них справедливо следуюшее соотношение:

$$
\left(h_{A} g, g^{\prime}\right)=\left(g, h_{A} g^{\prime}\right)+\left(\Phi_{A u}, \Phi_{u^{\prime}}\right)-\left(\Phi_{u}, \Phi_{A u^{\prime}}\right) .
$$

Так как пространства $\Re_{-1}(h)$ и $\Omega$ изоморфины и выполняются равенства $\left(\Phi_{A u}, \Phi_{u^{\prime}}\right)=$ $\left[A u, u^{\prime}\right]$ и $\left(\Phi_{u}, \Phi_{A u^{\prime}}\right)=\left[u, A u^{\prime}\right]$, где $[\cdot, \cdot]$ - скалярное произведение в $\Omega$, то соотношение (2.11) можно переписать в виде

$$
\left(h_{A} g, g^{\prime}\right)=\left(g, h_{A} g^{\prime}\right)+\left[A u, u^{\prime}\right]-\left[u, A u^{\prime}\right] .
$$

Воспользовавшись равенством (2.12), из последних рассуждений можно получить, что справедлива 
Лемма 2. Расширение $h_{A}$ оператора $h$, задаваемое с помощъю условия (2.10), является самосопряженным оператором тогда и только тогда, когда $A-$ самосопряженный оператор в $\Omega$.

Для тех элементов $g \in D\left(h^{*}\right)$, которым соответствуют достаточно быстро убывающие функции $u$ в разложении (2.8), определены асимптотики

$$
\int_{\left|k_{j}+\mu k_{i} / 2\right|<N} g\left(k_{1}, k_{2}\right) d k_{j}=(-1)^{j}\left[4 \pi u\left(k_{i}\right) N+G\left(k_{i}\right)+o(1)\right]
$$

при $N \rightarrow \infty$, где функция $G\left(k_{i}\right)$ имеет вид

$$
\begin{aligned}
G\left(k_{i}\right)= & -2 \pi^{2} \sqrt{\frac{1}{4}\left(4-\mu^{2}\right) k_{i}^{2}+1} u\left(k_{i}\right)-\int_{\mathbb{R}^{3}} \frac{u\left(k_{j}\right) d k_{j}}{k_{1}^{2}+\mu k_{1} k_{2}+k_{2}^{2}+1}+ \\
& +\frac{\pi^{2} v\left(k_{i}\right)}{\sqrt{\left(4-\mu^{2}\right) k_{i}^{2} / 4+1}}-\int_{\mathbb{R}^{3}} \frac{v\left(k_{j}\right) d k_{j}}{\left(k_{1}^{2}+\mu k_{1} k_{2}+k_{2}^{2}+1\right)^{2}}, \quad i, j=1,2, \quad i \neq j,
\end{aligned}
$$

и удовлетворяет условию (2.6). Обозначим через $h_{\varepsilon}^{*}$ сужение оператора $h^{*}$ на область $D\left(h_{\varepsilon}^{*}\right)$, состояшую из функций $g\left(k_{1}, k_{2}\right) \in D\left(h^{*}\right)$, которые удовлетворяют условию

$$
G(k)=-2 \pi^{2} \varepsilon u(k),
$$

где $\varepsilon$ - произвольная вешественная константа. Оператор $h_{\varepsilon}^{*}$ является сопряженным к некоторому симметрическому расширению $h_{\varepsilon}$ оператора $h$.

Рассмотрим семейство операторов $\{T(z), z \in \mathbb{C} \backslash[0, \infty)\}$ в $L_{2}\left(\mathbb{R}^{3}\right)$, действующих по формуле

$$
(T(z) u)\left(k_{1}\right)=2 \pi^{2} \sqrt{\frac{1}{4}\left(4-\mu^{2}\right) k_{1}^{2}-z} u(k)+\int_{\mathbb{R}^{3}} \frac{u\left(k_{2}\right)}{k_{1}^{2}+\mu k_{1} k_{2}+k_{2}^{2}-z} d k_{2}
$$

на множестве $D$ функций $u \in L_{2}\left(\mathbb{R}^{3}\right)$ таких, что

$$
|u(k)| \leqslant \frac{\text { const }}{1+|k|^{3}} .
$$

Далее введем в рассмотрение семейство операторов в $\Omega$

$$
A_{\varepsilon}=K^{-1}\left[\bar{T}(-1)-2 \pi^{2} \varepsilon I\right]
$$

где $\bar{T}(-1)$ - замыкание оператора $T(-1)$. Для любого $\varepsilon \in \mathbb{R}^{1}$ сопряженный оператор $A_{\varepsilon}^{*}$ определяется как

$$
A_{\varepsilon}^{*}=K^{-1}\left[T^{*}(-1)-2 \pi^{2} \varepsilon I\right], \quad D\left(A_{\varepsilon}^{*}\right)=D\left(T^{*}(-1)\right),
$$

где $T^{*}(-1)$ - оператор, сопряженный к $T(-1)$.

Из формул (2.13) и условия (2.14) видно, что если в формуле (2.10) оператор $A$ заменить на $A_{\varepsilon}^{*}$, то полученное сужение $h_{A_{\varepsilon}^{*}}$ оператора $h^{*}$ совпадает с оператором $h_{\varepsilon}^{*}$, т.е. 
$h_{\varepsilon}^{*}=h_{A_{\varepsilon}^{*}}$. Поскольку $h_{\varepsilon}^{*}=h_{A_{\varepsilon}^{*}}=\left(h_{A_{\varepsilon}}\right)^{*}$, то оператор $h_{\varepsilon}$ совпадает с оператором $h_{A_{\varepsilon}}$. Таким образом, симметрическое расширение $h_{\varepsilon}$ оператора $h$ получается сужением оператора $h^{*}$ на область $D\left(h_{A_{\varepsilon}}\right)=D\left(h_{\varepsilon}\right)$ с помошью условия $v=A_{\varepsilon} u$.

Применим выписанные соотношения для построения резольвенты оператора $h_{\varepsilon}$. Полагая $g=\left(h_{\varepsilon}-z I\right)^{-1} \psi$, из уравнения

$$
\left(h_{\varepsilon}-z E\right) g=\psi
$$

и формулы $(2.9)$ находим, что для любого $\psi \in L_{2}^{a}\left(\left(\mathbb{R}^{3}\right)^{2}\right)$ функция $g$ имеет вид

$$
g\left(k_{1}, k_{2}\right)=\frac{\psi\left(k_{1}, k_{2}\right)+u\left(k_{1}\right)-u\left(k_{2}\right)}{k_{1}^{2}+\mu k_{1} k_{2}+k_{2}^{2}-z} .
$$

Сравнивая (2.18) с (2.8) и используя условия $v=A_{\varepsilon} u$ и (2.4), мы приходим к уравнению для определения $u(k)$ через $\psi\left(k_{1}, k_{2}\right)$ :

$$
2 \pi^{2}\left(\sqrt{\frac{1}{4}\left(4-\mu^{2}\right) k^{2}-z}-\varepsilon\right) u(k)-\int_{\mathbb{R}^{3}} \frac{u\left(k^{\prime}\right)}{k^{2}+\mu k k^{\prime}+k^{\prime 2}-z} d k^{\prime}=\chi(k ; z),
$$

где

$$
\chi(k ; z) \equiv \int_{\mathbb{R}^{3}} \frac{\psi\left(k, k^{\prime}\right)}{k^{2}+\mu k k^{\prime}+k^{\prime 2}-z} d k^{\prime} .
$$

Заметим, что из леммы 2 и соотношений (2.8), (2.10), (2.16) и (2.17) следует, что самосопряженность оператора $h_{\varepsilon}$ равносильна сушественной самосопряженности оператора $T(-1)$, причем индексы дефекта оператора $T(-1)$ совпадают с индексами дефекта оператора $h_{\varepsilon}$.

\section{3. ОСНОВНЫЕ РЕЗУЛЬТАТЫ}

Обозначим через $\mu_{0}$ решение уравнения

$$
\pi^{2} \sqrt{4-\mu^{2}}+\frac{8 \pi}{\mu^{2}} \sqrt{4-\mu^{2}}\left(\arcsin \frac{\mu}{2}\right)-\frac{8 \pi}{\mu}=0, \quad \sqrt{3.47}<\mu_{0}<\sqrt{3.48}
$$

Tеорема 1. Если $\mu \in\left(\mu_{0}, 2\right)$, то оператор $\bar{T}(-1)$ имеет ненулевые, равные и конечные индексы дефекта.

ДоКАЗАТЕЛЬСТВо. Пусть $T$ - симметрический оператор, определенньй на области $D$ (см. (2.15)) и действующий по формуле

$$
(T u)(p)=\pi^{2} \sqrt{4-\mu^{2}}|p| u(p)-\int_{\mathbb{R}^{3}} \frac{u(q)}{p^{2}+\mu p q+q^{2}} d q .
$$

Так как прибавление ограниченного самосопряженного оператора не изменяет индексы дефекта и $T(-1)-T$ является ограниченным самосопряженным оператором, то вместо $T(-1)$ достаточно рассмотреть оператор $T$. 
Заметим, что оператор $T$ коммутирует с операторами представления группы вращений $O_{3}$ в $L_{2}\left(\mathbb{R}^{3}\right)$ :

$$
\left(V_{g} u\right)(p)=u\left(g^{-1} p\right), \quad u \in L_{2}\left(\mathbb{R}^{3}\right), \quad g \in O_{3},
$$

и приводится каждым подпространством $L_{l}$ функций вида

$$
u_{l}(p)=\sum_{m=-l}^{l} u_{m, l}(|p|) Y_{m}^{l}(\theta, \varphi)
$$

где $|p|=r, \theta, \varphi$ - сферические координаты вектора $p, Y_{m}^{l}(\theta, \varphi)$ - сферические функции веса $l$ (см. [8]), $u_{m, l}$ - функции, зависяшие от $|p|$. Часть оператора $T$, действуюшую в $L_{l}$, обозначим через $T_{l}$. Рассмотрим сначала оператор $T_{0}$, определенный в подпространстве $L_{0}$ сферически-симметричных функций. Выполняя в формуле (3.2) для сферически-симметричных функций интегрирование по углам, убедимся в том, что оператор $T_{0}$ унитарно-эквивалентен оператору $S_{0}$, действующему в пространстве $L_{2}\left(\mathbb{R}_{+}, r^{2} d r\right)$ по формуле

$$
\left(S_{0} u\right)(r)=\pi^{2} \sqrt{4-\mu^{2}} r u(r)+\frac{2 \pi}{\mu} \int_{0}^{\infty} \frac{t}{r} \ln \left(\frac{r^{2}+\mu r t+t^{2}}{r^{2}-\mu r t+t^{2}}\right) u(t) d t
$$

Область определения $D\left(S_{0}\right)$ оператора $S_{0}$ состоит из функций $u(r) \in L_{2}\left(\mathbb{R}_{+}, r^{2} d r\right)$, удовлетворяюших условию (2.15).

После унитарного в $L_{2}\left(\mathbb{R}_{+}, r^{2} d r\right)$ преобразования Меллина

$$
v(s)=\int_{0}^{+\infty} r^{-i s+1 / 2} u(r) d r
$$

где $u(r) \in D\left(S_{0}\right)$, оператор $S_{0}$ переходит в оператор

$$
\left(\widehat{S}_{0} v\right)(s)=\Psi_{0}\left(s+\frac{i}{2}\right) v(s+i)
$$

действуюший на области $D\left(\widehat{S}_{0}\right)$ функций $v(s)$ таких, что $v(s)$ аналитичны в полосе $0 \leqslant$ $\operatorname{Im} s \leqslant 1$ и $v(x+i \alpha) \in L_{2}\left(\mathbb{R}^{1}\right)$ для любого $\alpha \in[0,1]$. Здесь

$$
\Psi_{0}(s)=\pi^{2} \sqrt{4-\mu^{2}}+\frac{4 \pi^{2}}{\mu} \frac{\operatorname{sh}(\arcsin (\mu / 2)) s}{s \operatorname{ch}(\pi s / 2)} .
$$

Повторяя рассуждения, приведенные в работе [5], получим, что область определения $D\left(\widehat{S}_{0}^{*}\right)$ сопряженного оператора $\widehat{S}_{0}^{*}$ будет состоять из функций вида

$$
w(s)=\left[\Psi_{0}\left(s-\frac{i}{2}\right)\right]^{-1} v(s)
$$

где $v(s) \in D\left(\widehat{S}_{0}\right)$ (это легко выводится из теоремы братьев Рисс о мерах на окружности, ортогональных степеням (см. [9])). Из (3.6) видно, что функция $\Psi_{0}(s)$ аналитична и 
не имеет нулей в полосе $-1 \leqslant \operatorname{Im} s \leqslant 1$. Следовательно, функция $\left[\Psi_{0}(s-i / 2)\right]^{-1}$ аналитична и ограничена в полосе $0 \leqslant \operatorname{Im} s \leqslant 1$. Тогда из (3.7) видно, что все функции $w(s)$ из $D\left(\widehat{S}_{0}^{*}\right)$ аналитичны в полосе $0 \leqslant \operatorname{Im} s \leqslant 1$ и $|w(x+i \alpha)| \in L_{2}\left(\mathbb{R}^{1}\right)$ для любого $\alpha \in[0,1]$. Отсюда следует, что $D\left(\widehat{S}_{0}\right)=D\left(\widehat{S}_{0}^{*}\right)$, следовательно, $\widehat{T}_{0}$ - самосопряженный оператор. Таким образом, при всех значениях параметра $\mu$ оператор $\overline{T_{0}}$ является самосопряженным.

Рассмотрим теперь случай $l \geqslant 1$. Поскольку оператор с ядром $\left(p^{2}+\mu p q+q^{2}\right)^{-1}$ приводится каждым подпространством $L_{l}$, то имеет место разложение

$$
\frac{1}{p^{2}+\mu p q+q^{2}}=\sum_{l=0}^{\infty} a_{l}(r, t) \sum_{m=-l}^{l} Y_{l}^{m}(\theta, \varphi) Y_{l}^{m}\left(\theta^{\prime}, \varphi^{\prime}\right)
$$

где

$$
\begin{gathered}
p=(r \sin \theta \cos \varphi, r \sin \theta \sin \varphi, r \cos \theta), \quad q=\left(t \sin \theta^{\prime} \cos \varphi^{\prime}, t \sin \theta^{\prime} \sin \varphi^{\prime}, t \cos \theta^{\prime}\right), \\
a_{l}(r, t)=2 \pi \int_{-1}^{1} \frac{P_{l}(x)}{r^{2}+\mu x r t+t^{2}} d x, \quad P_{l}(x)=\frac{1}{2^{l} l !} \frac{d^{l}\left(x^{2}-1\right)^{l}}{d x^{l}} .
\end{gathered}
$$

Из (3.2), (3.3) и (3.8) следует, что оператор $T_{l}$ действует в $L_{l}$ следуюшим образом:

$$
\left(T_{l} u_{l}\right)(p)=\sum_{m=-l}^{l}\left(S_{l} u_{l, m}\right)(|p|) Y_{l}^{m}(\theta, \varphi)
$$

где $S_{l}$ - оператор в $L_{2}\left(\mathbb{R}_{+}, r^{2} d r\right)$, действующий на $D\left(S_{l}\right)=D\left(S_{0}\right)$ по формуле

$$
\left(S_{l} u\right)(r)=\pi^{2} \sqrt{4-\mu^{2}} r u(r)+\int_{0}^{\infty} t^{2} a_{l}(r, t) u(t) d t
$$

После преобразования (3.5) замыкание оператора $S_{l}$ перейдет в оператор

$$
\left(\widehat{S}_{l} w\right)(s)=\Psi_{l}\left(s+\frac{i}{2}\right) w(s+i)
$$

действуюший на области $D\left(\widehat{S}_{l}\right)=D\left(\widehat{S}_{0}\right)$, где

$$
\Psi_{l}(s)=\pi^{2} \sqrt{4-\mu^{2}}+2 \pi^{2} \int_{-1}^{1} P_{l}(x) \frac{\operatorname{sh}(\arccos (\mu x / 2)) s}{\sqrt{1-\mu^{2} x^{2} / 4} \operatorname{sh}(\pi s / 2)} d x .
$$

Рассуждая так же, как и в случае $l=0$, получим, что область определения $D\left(\widehat{S}_{l}^{*}\right)$ сопряженного оператора $\widehat{S}_{l}^{*}$ будет состоять из функций вида

$$
w(s)=\left[\Psi_{l}\left(s-\frac{i}{2}\right)\right]^{-1} v(s)
$$

где $v(s) \in D\left(\widehat{S}_{0}\right)$. 
С помощью несложных вычислений получим, что

$$
\Psi_{l}(s)=\pi^{2} \sqrt{4-\mu^{2}}-2 \pi^{2} \int_{0}^{1} P_{l}(x) \frac{\operatorname{sh}(\arcsin (\mu x / 2)) s}{\sqrt{1-\mu^{2} x^{2} / 4} \operatorname{sh}(\pi s / 2)} d x
$$

при $l=2 n-1, n=1,2,3, \ldots$,

$$
\Psi_{l}(s)=\pi^{2} \sqrt{4-\mu^{2}}+2 \pi^{2} \int_{0}^{1} P_{l}(x) \frac{\operatorname{ch}(\arcsin (\mu x / 2)) s}{\sqrt{1-\mu^{2} x^{2} / 4} \operatorname{ch}(\pi s / 2)} d x
$$

при $l=2 n, n=1,2,3, \ldots$.

Пусть $l=1$. Тогда

$$
\Psi_{1}(s)=\pi^{2} \sqrt{4-\mu^{2}}-2 \pi^{2} \int_{0}^{1} x \frac{\operatorname{sh}(\arcsin (\mu x / 2)) s}{\sqrt{1-\mu^{2} x^{2} / 4} \operatorname{sh}(\pi s / 2)} d x
$$

Очевидно, что для любого $\mu \in(0,2) \Psi_{1}(s)$ является четной и возрастаюшей по $|s|$ функцией, при этом

$$
\begin{gathered}
\lim _{|s| \rightarrow \infty} \Psi_{1}(s)=\pi^{2} \sqrt{4-\mu^{2}} \\
F_{1}(\mu)=\min \Psi_{1}(s)=\pi^{2} \sqrt{4-\mu^{2}}+\frac{8 \pi}{\mu^{2}} \sqrt{4-\mu^{2}}\left(\arcsin \frac{\mu}{2}\right)-\frac{8 \pi}{\mu} .
\end{gathered}
$$

Функция $F_{1}(\mu)$, определенная в интервале $(0,2)$ формулой $(3.15)$, непрерывна, убывает, и ее значения заполняют интервал $\left(-4 \pi, 2 \pi^{2}\right)$. Тогда уравнение $F_{1}(\mu)=0$ (или $(3.1)$ ) имеет единственное решение, которое мы обозначим через $\mu_{0}$.

Теперь перейдем к непосредственному доказательству утверждения теоремы 1 . Пусть $\mu \in\left(\mu_{0}, 2\right)$. Поскольку функция $F_{1}(\mu)$ непрерывна, убывает и $F_{1}\left(\mu_{0}\right)=0$, то при $\mu>\mu_{0} F_{1}(\mu)=\min \Psi_{l}(s)=\Psi_{1}(0)<0$. Отсюда в силу четности и монотонности по $|s|$ функции $\Psi_{1}(s)$ получим, что $\Psi_{1}(s)$ обрашается в нуль только в двух точках $s= \pm s_{0}$, где $s_{0}>0$ - простой корень уравнения $\Psi_{1}(s)=0$.

Из $\Psi_{1}\left( \pm s_{0}\right)=0$ следует, что $D\left(\widehat{S}_{1}^{*}\right)$ кроме элементов $D\left(\widehat{S}_{1}\right)$ включает в себя и функции

$$
\frac{1}{s-s_{0}-i / 2}, \quad \frac{1}{s+s_{0}-i / 2} .
$$

Это означает, что $D\left(\widehat{S}_{1}^{*}\right)$ состоит из элементов вида

$$
w(s)=\frac{c_{1}}{s-s_{0}-i / 2}+\frac{c_{2}}{s+s_{0}-i / 2}+v(s)
$$

где $v \in D\left(\widehat{S}_{1}\right)$. Таким образом, $D\left(\widehat{S}_{1}\right)$ в $D\left(\widehat{S}_{1}^{*}\right)$ имеет коразмерность 2 . Отсюда следует, что индексы дефекта оператора $\widehat{S}_{1}$ (и оператора $S_{1}$ ) равны либо $(2,0)$, либо $(0,2)$, либо $(1,1)$. Однако в силу вешественности оператора $S_{1}$ (см. (3.12)) его индексы дефекта одинаковы и равны $(1,1)$. Из $(3.3)$ и $(3.9)$ следует, что оператор $T_{1}$ также имеет индексы дефекта $(1,1)$. 
Процедура, аналогичная рассмотренной для случая $l=1$, покажет, что при $l \geqslant 2$ индексы дефекта оператора $T_{l}$ равны и не превышают $(1,1)$. Кроме того, для любого $\mu>\mu_{0}$ найдется натуральное число $l_{\mu}$ такое, что при $l \geqslant l_{\mu}$ индексы дефекта оператора $T_{l}$ равны $(0,0)$. Отсюда следует, что для любого $\mu \geqslant \mu_{0}$ оператор $T(-1)$ имеет равные и конечные индексы дефекта. Теорема 1 доказана.

Так как оператор $T_{1}(-1)$ получается из оператора $T_{1}$ прибавлением ограниченного самосопряженного оператора, то $T_{1}(-1)$ - симметрический оператор с индексами дефекта $(1,1)$. В доказательстве теоремы 1 фактически были описаны дефектные векторы операторов $T_{1}$ и $T_{1}(-1)$. Применив общую теорию Неймана самосопряженных расширений симметрических операторов (см. [3]), придем к семейству $\left\{T_{1 \beta}(-1)\right\}, \beta \in \mathbb{C}$, $|\beta|=1$, самосопряженных расширений оператора $T_{1}(-1)$. Описанию области определения оператора $T_{1 \beta}(-1)$ посвящается следуюшая теорема.

ТЕОрема 2. Область определения оператора $T_{1 \beta}(-1)$ состоит из элементов вида

$$
v(k)=c \frac{r^{i s_{0}}+\beta r^{-i s_{0}}}{r^{2}+1}+u(k),
$$

где $u \in D\left(\bar{T}_{1}(-1)\right), \quad r=|k| \in \mathbb{R}_{+}, \quad \beta \in \mathbb{C}^{1},|\beta|=1,-$ параметр самосопряжсенного расширения $T_{1 \beta}(-1)$ оператора $T_{1}(-1)$.

ДокАЗАТЕЛЬСтво. Из теоремы 1 следует, что дефектное пространство $\Re_{z}=$ $\left(\operatorname{Ran}\left(T_{1}(-1)-z I\right)\right)^{\perp}$ оператора $T_{1}(-1)$ одномерно, а из $(3.15)$ видно, что дефектный вектор $\theta_{z}(r) \in \Re_{z}$ оператора $T_{1}(-1)$ имеет вид

$$
\theta_{z}(k)=\frac{r^{i s_{0}}+\zeta r^{-i s_{0}}}{r^{2}+1}+w(k)
$$

где $\zeta$ - некоторое комплексное число, а $w \in D\left(\bar{T}_{1}(-1)\right)$. Следовательно,

$$
\theta_{\bar{z}}(k)=\overline{\theta_{z}(k)}=\frac{\bar{\zeta} r^{i s_{0}}+r^{-i s_{0}}}{r^{2}+1}+\overline{w(k)}
$$

с точностью до умножения на константу.

Рассмотрим произвольное самосопряженное расширение $\widehat{T}_{1}(-1)$ оператора $T_{1}(-1)$. Поскольку $\left\|\theta_{\bar{z}}\right\|=\left\|\theta_{z}\right\|$, из формул Неймана следует, что область определения $D\left(\widehat{T}_{1}(-1)\right)$ оператора $\widehat{T}_{1}(-1)$ состоит из элементов вида

$$
v(k)=\theta_{z}(k)+\hat{\beta} \theta_{\bar{z}}(k)+u_{1}(k)
$$

где $u_{1} \in D\left(\bar{T}_{1}(-1)\right), \theta_{z} \in \Re_{z}$, для некоторой константы $\hat{\beta} \in \mathbb{C}^{1},|\hat{\beta}|=1$, характеризуюшей расширение $\widehat{T}_{1}(-1)$. Таким образом, с точностью до умножения на константу элемент из $D\left(\widehat{T}_{1}(-1)\right)$ будет иметь вид

$$
v(k)=\left(r^{i s_{0}}+\frac{\zeta+\hat{\beta}}{1+\bar{\zeta} \hat{\beta}} r^{-i s_{0}}\right)\left(r^{2}+1\right)^{-1}+u(k),
$$


где $u \in D\left(\bar{T}_{1}(-1)\right)$. Легко заметить, что если обозначить $\beta=(\zeta+\hat{\beta}) /(1+\bar{\zeta} \hat{\beta})$, то $|\beta|=1$ для $\zeta \in \mathbb{C}^{1}, \hat{\beta} \in \mathbb{C}^{1},|\hat{\beta}|=1$. Но отсюда следует утверждение теоремы (если принять обозначение $\left.\widehat{T}_{1}(-1)=T_{1 \beta}(-1)\right)$.

Пусть $\mu_{0}<\mu<2$. В этом случае исследуем оператор $h_{\varepsilon}$.

Легко видеть, что оператор $h_{\varepsilon}$ коммутирует с операторами группы врашений $O_{3}$ в $L_{2}^{a}\left(\left(\mathbb{R}^{3}\right)^{2}\right)$ :

$$
\left(U_{g} \psi\right)\left(k_{1}, k_{2}\right)=\psi\left(g^{-1} k_{1}, g^{-1} k_{2}\right), \quad \psi \in L_{2}^{a}\left(\left(\mathbb{R}^{3}\right)^{2}\right), \quad g \in O_{3},
$$

и, следовательно, он приводится каждым подпространством $\aleph_{l}, l=0,1,2, \ldots$, функций вида

$$
\psi_{l}\left(k_{1}, k_{2}\right)=\sum_{k, m=-l}^{l} \psi_{l}^{k, m}\left(\left|k_{1}\right|,\left|k_{2}\right|\right) Y_{k}^{l}\left(\theta_{1}, \varphi_{1}\right) Y_{m}^{l}\left(\theta_{2}, \varphi_{2}\right),
$$

где $\psi_{l}^{k, m}$ - функция из $L_{2}\left(\left(\mathbb{R}_{+}\right)^{2}, r^{2} t^{2} d r d t\right) \equiv L_{2}\left(\mathbb{R}_{+}, r^{2} d r\right) \otimes L_{2}\left(\mathbb{R}_{+}, t^{2} d t\right), \quad Y_{k}^{l}$ и $Y_{m}^{l}$ - сферические функции с угловым моментом $l,\left|k_{j}\right|, \theta_{j}, \varphi_{j}$ - сферические координаты вектора $p_{j}, j=1,2$.

Часть оператора $h_{\varepsilon}$, действуюшую в $\aleph_{l}$, обозначим через $h_{\varepsilon}(l)$. Подпространство $\aleph_{0}$ состоит из функций $\psi\left(k_{1}, k_{2}\right)$, не меняюшихся при врашениях (3.19). В силу теоремы 1 оператор $h_{\varepsilon}(0)$ является самосопряженным. Далее часть оператора $h^{*}$, действуюшую в $\aleph_{l}$, обозначим через $h_{l}^{*}$.

Пусть $\Omega_{l}, l=0,1,2, \ldots,-$ подпространство функций $u \in \Omega$, получаюшееся пополнением подпространства $L_{l}$ по скалярному произведению $[u, v]=(K u, v)$. Часть оператоpa $A_{\varepsilon}$, действуюшую в $\Omega_{l}$, обозначим через $A_{\varepsilon}(l)$, а часть оператора $K$, действуюшую в $L_{l}$, через $K_{l}$. Тогда оператор $A_{\varepsilon}(l)$ можно определить следуюшим образом:

$$
A_{\varepsilon}(l)=K_{l}^{-1}\left[\bar{T}_{l}(-1)-2 \pi^{2} I\right], \quad D\left(A_{\varepsilon}(l)\right)=D\left(\bar{T}_{l}(-1)\right) \quad\left(\subset \Omega_{l}\right) .
$$

Нетрудно убедиться в том, что область определения $D\left(h_{l}^{*}\right)$ оператора $h_{l}^{*}$ состоит из таких функций $g\left(k_{1}, k_{2}\right)$ вида $(2.8)$, для которых $f \in D(h) \cap \aleph_{l}, u, v \in \Omega_{l}$. Аналогично область определения $D\left(h_{\varepsilon}(l)\right)$ оператора $H_{\varepsilon}(l)$ состоит из таких функций $g\left(k_{1}, k_{2}\right)$ вида (2.8), для которых $f \in D(h) \cap \aleph_{l}, u, v \in \Omega_{l}$ и $v=A_{\varepsilon}(l) u$.

Лемма 3. Оператор $A_{\varepsilon}$ является симметрическим, его индексы дефекта равны и совпадают с индексами дефекта оператора $T(-1)$. В частности, $A_{\varepsilon}(0)$ самосопряженный оператор, а $A_{\varepsilon}(1)$ - симметрический оператор с индексами дефекта $(1,1)$.

Доказательство леммы следует из того факта, что квадратичная форма оператора $A_{\varepsilon}$ в $\Omega$ совпадает с квадратичной формой оператора $\bar{T}(-1)-2 \pi^{2} I$ в $L_{2}\left(\mathbb{R}^{3}\right)$. Действительно,

$$
\left[A_{\varepsilon} u, v\right]=\left(K K^{-1}\left[\bar{T}(-1)-2 \pi^{2} I\right] u, v\right)=\left(\left[\bar{T}(-1)-2 \pi^{2} I\right] u, v\right),
$$

где $u, v \in D(\bar{T}(-1))$.

Рассмотрим семейство $\left\{A_{\varepsilon \beta}(1)\right\}, \varepsilon \in \mathbb{R}^{1}, \beta \in \mathbb{C}^{1},|\beta|=1$, операторов

$$
A_{\varepsilon \beta}(1)=K_{1}^{-1}\left[T_{1 \beta}(-1)-2 \pi^{2} I\right], \quad D\left(A_{\varepsilon \beta}(1)\right)=D\left(T_{1 \beta}(-1)\right),
$$


действуюших в подпространстве $\Omega_{1}$. Поскольку квадратичная форма $\left[A_{\varepsilon}(1) u, v\right]$ в $\Omega_{1}$ совпадает с квадратичной формой $\left(\left[T_{1 \beta}(-1)-2 \pi^{2} I\right] u, v\right)$ в $L_{1} \subset L_{2}\left(\mathbb{R}^{3}\right)$, то из самосопряженности оператора $T_{1 \beta}(-1)$ вытекает самосопряженность оператора $A_{\varepsilon}(1)$. Обозначим через $h_{\varepsilon \beta}(1)$ сужение оператора $h_{1}^{*}$ на область $D\left(h_{\varepsilon \beta}(1)\right)$, состояшую из таких функций $g\left(k_{1}, k_{2}\right)$ вида $(2.8)$, для которых $f \in D(h) \cap \aleph_{1}, u, v \in \Omega_{1}$ и $v=A_{\varepsilon}(1) u$. $\mathrm{C}$ помошью формулы (2.17) можно установить, что для любых $\varepsilon \in \mathbb{R}^{1}$ и $\beta \in \mathbb{C}^{1},|\beta|=1$, оператор $h_{\varepsilon \beta}(1)$ является самосопряженным.

Лемма 4. Для любого $\varepsilon \in \mathbb{R}^{1}$ симметрический оператор $h_{\varepsilon}$ имеет ненулевье, равные и конечные индексы дефекта. В частности, оператор $h_{\varepsilon}(1)$ имеет индексы дефекта $(1,1)$.

ДокАЗАТЕЛЬСТВо. Из обшей теории самосопряженных расширений симметрических операторов легко следует (см. [3]) следующее

ПРЕДЛОЖЕНИЕ. Коразмерность области определения $D(A)$ симметрического оператора $A$ с конечными и равными индексами дефекта $(n, n)$ в области $D(\hat{A})$ любого его самосопряженного расширения равна $n$. Если индексы дефекта оператора А бесконечны и у него существуют самосопряэсенные расширения, то найдется такое самосопряженное расширение $\hat{A}$, что $D(A)$ имеет в $D(\hat{A})$ бесконечную коразмерность.

Вернемся к схеме расширения операторов $h_{\varepsilon}$ и $h_{\varepsilon}(1)$. Все самосопряженные расширения оператора $h_{\varepsilon}(1)$ являются самосопряженными расширениями оператора $A_{\varepsilon}(1)$, поэтому они описываются введенным выше классом операторов $h_{\varepsilon}(1)$. В силу предложения коразмерность $D\left(A_{\varepsilon}(1)\right)$ в $D\left(A_{\varepsilon \beta}(1)\right)\left(D\left(T_{1}(-1)\right)\right.$ в $\left.D\left(T_{1 \beta}(-1)\right)\right)$ равна единице. Отсюда и из схем расширения оператора $h_{\varepsilon}(1)$ следует, что коразмерность области определения $D\left(h_{\varepsilon}(1)\right)$ оператора $h_{\varepsilon}(1)$ в области определения $D\left(h_{\varepsilon \beta}(1)\right)$ оператора $h_{\varepsilon \beta}(1)$ равна единице. Теперь утверждение леммы следует из предложения.

Как и в случае $l=1$, можно показать, что индексы дефекта операторов $h_{\varepsilon}(l), l \geqslant 1$, равны и не превышают $(1,1)$. Для любого $\mu>\mu_{0}$ найдется натуральное число $l_{\mu}$ такое, что при $l>l_{\mu}$ все операторы $h_{\varepsilon}(l)$ являются самосопряженными. Таким образом, для любого $\varepsilon \in \mathbb{R}^{1}$ оператор $h_{\varepsilon}$ является симметрическим оператором с ненулевыми, равными и конечными индексами дефекта. Лемма доказана.

Основным результатом данной работы является следуюшая

ТЕорема 3. Любое самосопряженное расширение $\hat{h}_{\varepsilon}$ оператора $h_{\varepsilon}$ имеет бесконечный набор отрицательных собственных значений, уходящих на $-\infty$.

Нижеследующие леммы представляют собой этапы доказательства этой теоремы.

Заметим, что если $\hat{h}_{\varepsilon}$ - некоторое самосопряженное расширение оператора $h_{\varepsilon}$, то сушественньй спектр оператора $\hat{h}_{\varepsilon}$ заполняет отрезок $[0,+\infty)$ при $\varepsilon<0$ и отрезок $\left[-\varepsilon^{2},+\infty\right)$ при $\varepsilon>0$.

Из леммы 4 следует, что для любого $z \in \mathbb{C}^{1} \backslash \mathbb{R}^{1}$ сушествует единственное с точностью до умножения на константу решение уравнения

$$
\left(h_{\varepsilon}^{*}(1)\right) g=z g .
$$


Из (2.8) с дополнительными условиями $f \in D(h) \cap \aleph_{1}, u, v \in \Omega_{1}, v=A_{\varepsilon}^{*}(1) u$, учитывая определение $A_{\varepsilon}(1)$, получим, что решение уравнения (3.21) имеет вид

$$
\begin{aligned}
g\left(k_{1}, k_{2}\right)= & f\left(k_{1}, k_{2}\right)+\frac{\sum_{m=-1}^{1}\left[u_{m}\left(r_{1}\right) Y_{1}^{m}\left(\theta_{1}, \varphi_{1}\right)-u_{m}\left(r_{2}\right) Y_{1}^{m}\left(\theta_{2}, \varphi_{2}\right)\right]}{k_{1}^{2}+\mu k_{1} k_{2}+k_{2}^{2}+1}+ \\
& +\frac{\sum_{m=-1}^{1}\left[\left(A_{\varepsilon}^{*}(1) u_{m}\right)\left(r_{1}\right) Y_{1}^{m}\left(\theta_{1}, \varphi_{1}\right)-\left(A_{\varepsilon}^{*}(1) u_{m}\right)\left(r_{2}\right) Y_{1}^{m}\left(\theta_{2}, \varphi_{2}\right)\right]}{\left(k_{1}^{2}+\mu k_{1} k_{2}+k_{2}^{2}+1\right)^{2}},
\end{aligned}
$$

где $A_{\varepsilon}^{*}(1)$ - оператор, сопряженньй к оператору $A_{\varepsilon}(1)$. Но из $(2.9)$ и $(3.21)$ следует, что решение уравнения (3.21) имеет вид

$$
g\left(k_{1}, k_{2}\right)=\frac{\sum_{m=-1}^{1}\left[u_{m}\left(r_{1}\right) Y_{1}^{m}\left(\theta_{1}, \varphi_{1}\right)-u_{m}\left(r_{2}\right) Y_{1}^{m}\left(\theta_{2}, \varphi_{2}\right)\right]}{k_{1}^{2}+\mu k_{1} k_{2}+k_{2}^{2}-z} .
$$

Сравнивая (3.22) с (3.23) и пользуясь определением оператора $A_{\varepsilon}^{*}(1)$, получим, что функции $u_{m}(r)$ являются решениями уравнения

$$
\left(\sqrt{\frac{1}{4}\left(4-\mu^{2}\right) r^{2}-z}-\varepsilon\right) u(r)+\frac{1}{\pi} \int_{0}^{\infty} t^{2}\left(\int_{-1}^{1} \frac{x d x}{r^{2}+\mu x r t+t^{2}-z}\right) u(t) d t=0
$$

Далее, вычислив интеграл

$$
\int_{-1}^{1} \frac{x d x}{r^{2}+\mu x r t+t^{2}-z} u(t) d t
$$

приходим к уравнению

$$
\begin{aligned}
& 2 \pi^{2}\left(\sqrt{\left.\frac{1}{4}\left(4-\mu^{2}\right) r^{2}-z-\varepsilon\right) u(r)+}\right. \\
& \quad+2 \pi \int_{0}^{\infty} \frac{t}{\mu r}\left[2-\frac{r^{2}+t^{2}-z}{\mu r t} \ln \left(\frac{r^{2}+t^{2}-z+\mu r t}{r^{2}+t^{2}-z-\mu r t}\right)\right] u(t) d t=0 .
\end{aligned}
$$

ЛЕмма 5. Если $\lambda_{0}>2 \varepsilon$, то при $z=-\lambda_{0}^{2}$ уравнение (3.24) имеет решение.

ДокаЗАТЕЛЬСтво. Выберем $z=-\lambda_{0}^{2}+i \delta$, где $\delta>0$ и мало́. При таких $z$ решение системы (3.25) сушествует, и из представления

$$
\begin{aligned}
u(r)= & \frac{1}{\pi \mu}\left(\sqrt{\frac{1}{4}\left(4-\mu^{2}\right) r^{2}+\lambda_{0}^{2}-i \delta}-\varepsilon\right)^{-1} \times \\
& \times \int_{0}^{\infty} \frac{t}{r}\left[-2+\frac{r^{2}+t^{2}+\lambda_{0}^{2}-i \delta}{\mu r t} \ln \left(\frac{r^{2}+\mu r t+t^{2}+\lambda_{0}^{2}-i \delta}{r^{2}-\mu r t+t^{2}+\lambda_{0}^{2}-i \delta}\right)\right] u(t) d t
\end{aligned}
$$

следует, что $u(r)$ аналитична в максимальной угловой окрестности положительной вешественной полуоси, ограниченной лучами, проходящими через начало координат и точки $z_{0}$ и $\overline{z_{0}}$, где $z_{0}^{2}=4\left(\varepsilon-\lambda_{0}^{2}+i \delta\right) /\left(4-\mu^{2}\right.$ ) (это область, в которой разность, стоящая в знаменателе в правой части (3.26), не обрашается в нуль). 
Сделав в (3.26) замену $r=\sqrt{-z} \zeta, t=\sqrt{-z} \xi$, получим уравнение

$$
\begin{aligned}
u(\sqrt{-z} \zeta)= & \frac{1}{\pi \mu}\left(\sqrt{\frac{1}{4}\left(4-\mu^{2}\right) r^{2}+1}-\frac{\varepsilon}{\sqrt{-z}}\right)^{-1} \times \\
& \times \int_{\gamma_{\xi}} \frac{\xi}{\zeta}\left[-2+\frac{\zeta^{2}+\xi^{2}+1}{\mu \zeta \xi} \ln \left(\frac{\zeta^{2}+\mu \zeta \xi+\xi^{2}+1}{\zeta^{2}-\mu \zeta \xi+\xi^{2}+1}\right)\right] u(\sqrt{-z} \xi) d \xi
\end{aligned}
$$

(здесь выбрана ветвь $\operatorname{Im} \sqrt{-z}>0$ ). Заметим, что согласно выбору $z$ контур $\gamma_{\xi}$ есть луч, лежащий в области аналитичности функции $u(\xi)$. Ввиду того, что интеграл в правой части (3.27) по дуге окружности, соединяющей контур $\gamma_{\xi}$ и вешественную положительную полуось, стремится к нулю с ростом радиуса, интегрирование можно проводить по вешественной полуоси. Кроме того, из представления (3.27) следует существование предела $u(\sqrt{-z} \zeta)$ при выходе $\zeta$ на вешественную ось. Переходя теперь к случаю $\delta=0$ (что возможно в силу аналитичности правой части (3.27) по $z$ ), получим сушествование решений уравнения

$$
\begin{aligned}
u\left(\lambda_{0} \zeta\right)= & \frac{1}{\pi \mu}\left(\sqrt{\frac{1}{4}\left(4-\mu^{2}\right) r^{2}+1}-\frac{\varepsilon}{\sqrt{-z}}\right)^{-1} \times \\
& \times \int_{\gamma_{\xi}} \frac{\xi}{\zeta}\left[-2+\frac{\zeta^{2}+\xi^{2}+1}{\mu \zeta \xi} \ln \left(\frac{\zeta^{2}+\mu \zeta \xi+\xi^{2}+1}{\zeta^{2}-\mu \zeta \xi+\xi^{2}+1}\right)\right] u\left(\lambda_{0} \xi\right) d \xi
\end{aligned}
$$

Сделав в (3.28) замену $r=\lambda_{0} \zeta, t=\lambda_{0} \xi$, придем к утверждению леммы.

СлЕДСТВИЕ. Уравнение (3.25) при $z=-\lambda^{2}, \lambda>\lambda_{0}$, имеет решение, которое можно получить из решения этого уравнения при $z=-\lambda_{0}^{2}, \quad \varepsilon^{\prime}=\left(\lambda_{0} / \lambda\right) \varepsilon$ заменой переменных $r \rightarrow\left(\lambda / \lambda_{0}\right) r, \quad t \rightarrow\left(\lambda / \lambda_{0}\right) t$.

Заметим, что решения уравнения (3.25) лежат в области определения $D\left(T_{1}^{*}(-1)\right)$ оператора $T_{1}^{*}(-1)$. Вернувшись к описанию (3.17) этой области и выполнив обратное преобразование Меллина, получим, что имеет место представление (с точностью до умножения на константу) решения уравнения (3.25) в виде

$$
u(r)=\frac{r^{i s_{0}}}{r^{2}+1}+\frac{c(\varepsilon) r^{-i s_{0}}}{r^{2}+1}+\tilde{u}(r)
$$

где $\tilde{u} \in D\left(\bar{T}_{0}(-1)\right), c(\varepsilon)$ - константа $(|c(\varepsilon)|=1$ в силу вешественности решения).

ЛЕмма 6. Функиия $c(\varepsilon)$ непрерывна в нуле.

ДокАЗАТЕЛЬСтво. Из представления (3.26) следует, что $u(r)$ непрерывна по $\varepsilon$. Следовательно, ее преобразование Меллина также непрерывно по $\varepsilon$. Но оно имеет вид (3.17), и функция $c(\varepsilon)$ есть вычет в точке $s_{0}+i / 2$ преобразования Меллина $g(s)$ функции $u(r)$ и, следовательно, непрерывна по $\varepsilon$. Лемма доказана.

Приступим к непосредственному доказательству теоремы 3 . Обозначим через $\hat{h}_{\varepsilon}(l)$ часть оператора $\hat{h}_{\varepsilon}$, действующую в подпространстве $\aleph_{l}$. Так как для любого $\mu \in(0,2)$ оператор $h_{\varepsilon}(0)$ является самосопряженным, то оператор $h_{\varepsilon}(0)$ равен $\hat{h}_{\varepsilon}(0)$ и, следовательно, оператор $\hat{h}_{\varepsilon}(0)$ не имеет собственного значения левее сушественного спектра 
оператора $\hat{h}_{\varepsilon}$. Оператор $\hat{h}_{\varepsilon}(1)$ является самосопряженным расширением для оператоpa $h_{\varepsilon}(1)$. Поскольку все самосопряженные расширения оператора $h_{\varepsilon}(1)$ исчерпьваются семейством $\left\{h_{\varepsilon \beta}(1), \beta \in \mathbb{C}^{1},|\beta|=1\right\}$, то найдется некоторое значение $\hat{\beta},|\hat{\beta}|=1$, такое, что $h_{\varepsilon \hat{\beta}}(1)=\hat{h}_{\varepsilon}(1)$. Поэтому достаточно исследовать оператор $h_{\varepsilon \hat{\beta}}(1)$.

В силу теоремы 2 область определения $D\left(T_{1, \hat{\beta}}(-1)\right)$ оператора $T_{1, \hat{\beta}}(-1)$ состоит из функций вида

$$
v(k)=\frac{r^{i s_{0}}+\hat{\beta} r^{-i s_{0}}}{r^{2}+1}+u(k), \quad u(k) \in D\left(\bar{T}_{1}(-1)\right) .
$$

Найдем теперь собственную функцию оператора $h_{\varepsilon \hat{\beta}}(1)$, отвечающую собственному значению $-\lambda^{2}$. Нетрудно видеть, что ее можно получить в виде (2.8) с учетом условия $v=A_{\varepsilon, \hat{\beta}}(1) u$ из решения $u(k)$ уравнения (3.26) при $z=-\lambda^{2}$ с дополнительным условием $u(k) \in D\left(T_{1, \hat{\beta}}(-1)\right)$. Это значит, что функция $v(k)$ должна удовлетворять представлению (3.30).

В силу леммы 4 и следствия леммы 5 при достаточно больших $\lambda$ условие (3.30) равносильно условию

$$
c(0)\left(\frac{\lambda}{\lambda_{0}}\right)^{2 i s_{0}}=\hat{\beta},
$$

где $c(0)=\lim _{\varepsilon \rightarrow 0} c(\varepsilon), \quad \lambda_{0}-$ некоторое фиксированное число, при котором уравнение (3.25) с $z=-\lambda_{0}^{2}$ имеет решение. Но условию (3.31) удовлетворяет бесконечный набор значений

$$
\lambda_{n}=\lambda_{0} \exp \left(\frac{\arg (\hat{\beta} / c(0))}{2 s_{0}}+\frac{\pi n}{s_{0}}\right), \quad n=0,1,2, \ldots
$$

Таким образом, для оператора $\hat{h}_{\varepsilon}$ мы получили бесконечный набор собственных значений $z_{n}=-\lambda_{n}^{2}$, уходящих на $-\infty$. Теорема 3 доказана.

Благодарности. Автор выражает особую признательность Р. А. Минлосу за постановку задачи и ценные советы. Работа частично поддержана Фондом фундаментальных исследований АН РУз.

\section{Список литературы}

[1] Р. А. Минлос, Л. Д. Фаддеев. ДАН СССР. 1961. Т. 141. № 6. С. 1335-1338.

[2] Р. А. Минлос, Л. Д. Фаддеев. ЖЭТФ. 1961. Т. 41. № 12. С. 1850-1851.

[3] Н. И. Ахиезер, И. М. Глазман. Теория линейных операторов в гильбертовом пространстве. М.: Наука, 1966.

[4] Р. А. Минлос, М. Х. Шерматов. Вестн. МГУ. Сер. 1. 1989. № 6. С. 7-14.

[5] A. M. Mel'nikov, R. A. Minlos. Adv. Sov. Math. 1991. V. 5. P. 99-112.

[6] М. Ш. Бирман. Матем. сб. 1956. Т. 38. № 4. С. 431-450.

[7] М. Х. Шерматов. ТМФ. 2000. Т. 125. № 1. С. 74-91.

[8] И. М. Гельфанд, Р. А. Минлос, З. Я. Шапиро. Представление группы врашений и группы Лоренца, их применения. М.: Физматгиз, 1958.

[9] К. Гофман. Банаховы пространства аналитических функций. М.-Л.: ИЛ, 1963.

Поступила в редакцию 4.IX.2002 г. 